\title{
Los proyectos de aula:
}

\section{una experiencia pedagógica con la vida en el circo}

\section{Classroom Projects: A Pedagogical Experience with Life in the Circus}

\section{Profesoras de preescolar del Instituto Pedagógico Nacional}

\section{Resumen}

Esta experiencia es fruto de la sistematización del proyecto de aula desarrollado en el colegio Instituto Pedagógico Nacional en el año 2005, llamado "La vida en el circo". El trabajo se enmarca en un contexto de constante esfuerzo de las maestras por la vinculación de la escuela a estrategias innovadoras por medio de las cuales se dé vida al aprendizaje y la enseñanza, de manera significativa, tanto para los niños como para las maestras. Dentro de los aspectos destacados como fruto de la reflexión pedagógica se encuentran principios que configuran la planeación, desarrollo y socialización, actores protagonistas, y ejes centrales de esta propuesta.

\section{Palabras clave}

Proyectos de aula, planeación flexible, socialización, práctica pedagógica, experiencias significativas, saberes pedagógicos.

\section{Summary}

This experience is the product of the systematization of a classroom project called "Life in the Circus" carried out in Instituto Pedagógico Nacional in 2005. The work is developed in a context in which a continuous effort done by the teachers allows the integration at school of new strategies that boost knowledge and learning significantly for children and teachers. Regarding relevant issues as part of the pedagogical reflection, it is found principles that constitute planning, carrying out and socialization, the main guidelines for this proposal.

\section{Key words}

Classroom projects, flexible planning, socialization, pedagogical practice, significant experiences, academic knowledge. 
En el siguiente artículo se exponen las reflexiones que han surgido de la experiencia con los niños de 5 a 6 años, grupo de Transición, en el Instituto Pedagógico Nacional. A partir del trabajo por proyectos de aula, las maestras de este nivel iniciamos una labor que nos permitió reconstruir de forma escrita el proceso de planeación, desarrollo y socialización del proyecto "La vida en el circo", para mejorar nuestra práctica y poder compartir esta experiencia con otros maestros que también están dispuestos a rescatar y enriquecer su quehacer pedagógico con esta estrategia, que aprovecha las múltiples riquezas que se encuentran al compartir con las diferentes disciplinas y la familia.

\section{Un trabajo gratificante}

Este ejercicio escritural, realizado con el apoyo y motivación de la Red de Cualificación de Educadores en Ejercicio (RED-CEE), recoge la experiencia vivida de manera sistemática para poder compartirla con otros, configurando una comunidad académica, que permitió identificar que la construcción histórica de la práctica pedagógica se ha gestado de manera empírica y se complementa con elementos del enfoque constructivista (Garton, 1994). Inicialmente se retomaron componentes piagetianos para retroalimentarlos con la teoría soci ocultural de Vigotsky, en búsqueda de respuestas y orientaciones a las dudas que surgieron en la práctica. De esta manera se fortaleció nuestra capacidad personal y de grupo, dando lugar a nuevos interrogantes, posibilidades y a la clarificación de saberes pedagógicos respecto a las prácticas desarrolladas. Esta experiencia pretendió trascender a otros maestros de la misma institución, generando reflexiones para mejorar el quehacer del docente.

Se conformó un verdadero equipo con las compañeras del nivel, quienes motivadas con nuevas ideas hicieron que el proyecto se caracterizara por su cooperativismo, energía y entusiasmo. "La vida en el circo" como proyecto de aula ha movido nuestros pensamientos, deseos y motivaciones, sin perder de vista la particularidad del grupo; descubrimos que cada una tiene un motor que podía generar continuamente multiplicidad de ideas que representaban oportunidades en el aula como las mejores y más significativas experiencias de aprendizaje. Hoy reconocemos que cuando las experiencias de vida llegan hasta lo más profundo de nosotras mismas, es posible transformar la actitud, enriquecer los planes y la vida en el aula.

Repensar en el quehacer como maestras para analizar desde allí las prácticas nos brindó herramientas para hacer conciencia sobre la concepción que se evidenciaba de los proyectos, nos permitió descubrir elementos favorables como el interés hacia un tema, la integración de los espacios disciplinares específicos, la planeación y el desarrollo, siempre con base en la motivación y los intereses del grupo, donde cada actividad nos permitía fortalecerlos y redimensionarlos, posibilitando una retroalimentación de las experiencias vividas, recogiendo constantemente los logros, los alcances, las fortalezas, las dificultades, para estar en permanente proceso evaluativo del trabajo pedagógico.

\section{Todo empezó así}

En la institución todos los maestros de preescolar y primaria tenemos un encuentro semanal de nivel (están presentes las directoras de curso y los profesores de las otras áreas), en el cual se socializan tareas por hacer, se definen estrategias y propuestas, se reconocen las fortalezas y las debilidades, se plantean sugerencias, se pactan acuerdos, se organizan actividades y salidas en forma conjunta para estar en permanente proceso evaluativo del trabajo pedagógico.

Durante uno de estos encuentros semanales empezamos a identificar las necesidades y proyecciones del grupo para 2005, esto nos llevó a plantear una estrategia que permitiera el trabajo en equipo, que fortaleciera el apoyo permanente y compartir experiencias individuales que enriquecieran el trabajo colectivo. Se pensó entonces en retomar los proyectos de aula como una herramienta que permite rescatar los intereses de los niños y de los maestros en un ambiente de participación y de permanente planeación y revisión; se hicieron ajustes, cambios que le dieran fortaleza durante su desarrollo eligiendo una temática unificada y desde allí compartir las experiencias de cada salón para retroalimentar el trabajo; ya que todas no teníamos la misma experiencia, surgía como necesidad fortalecerla y a nivel institucional crear impacto y hacer visible el desarrollo del proyecto.

Las maestras del nivel realizamos una lluvia de ideas (léase listado de intereses) sobre las posibles temáticas 
del proyecto para ese año. Al ir planteando las propuestas, se iba haciendo una recapitulación retrospectiva de las experiencias, evocando el interés por los medios audiovisuales (video, fotografía) que complementan y registran dejando una huella histórica de lo trabajado. Fue allí donde se abordaron las experiencias que cada una había vivido con esta metodología, y encontramos muchas riquezas en estos relatos. Uno de ellos, llamado "Los dinosaurios", era del año 1988, el cual nació a partir del interés suscitado en los niños por la película Jurasic Park. Con este proyecto se gestó el cambio en la metodología tradicional que aplicaban las maestras en el aula de clase, se observó que los niños aprendían con mayor facilidad, y eran interesantes y significativos en su cotidianidad.

Otros proyectos fueron "Los osos", que en su desarrollo se transformó en "Los osos y sus amigos"; "Así es Bogotá", que motivó el estudio y la profundización de "Los muiscas", "El aeropuerto", "Los insectos", "El sistema solar", "Soy parte de una linda familia", entre otros. Algunos de ellos se han socializado en ámbitos extraescolares como colegios, foros internacionales, Ministerio de Educación Nacional. Pero hubo uno que nos llamó la atención: "El circo", realizado en el año 2002, puesto que los niños demostraron facultad para expresarse espontáneamente; fue un proyecto rico en elementos como disfraces, historias, vivencias, en el cual se evidenció que no solo se divirtieron los niños, sino también lo disfrutaron las maestras por toda la magia y la fantasía que contenía. Este proyecto lo realizó el curso kínder y se dio a conocer a los otros niños del nivel mostrando los disfraces y lo que habían elaborado. A partir de allí nos proyectamos, puesto que generó gran afinidad para las maestras y los niños.

\section{El deseo y el interés...}

En este momento de socialización se encontró que todas las experiencias evocaban recuerdos maravillosos, como la participación activa de los niños, el trabajo en equipo familia-escuela, que, además de brindarnos un momento de esparcimiento, ayudaron a descubrir un elemento determinante para elegir cuál sería el proyecto de aula; era primordial que la temática fuera interesante y muy significativa para los niños, pero al mismo tiempo para las maestras, puesto que en su desarrollo nos sentimos parte del proceso y al mismo tiempo investigamos sobre los temas a través de videos, documentales y consultamos bibliografía; así aprendimos y nos divertimos. Fue así como se decidió "La vida en el circo" como una fuente de magia, alegría, color, que para los niños representó diversión, asombro y en nosotras evocó recuerdos agradables que brindaron diferentes posibilidades pedagógicas.

El proyecto se inició en marzo de 2005, con los grados de transición (niños entre 5 y 6 años). A partir del día a día se fueron identificando algunos principios pedagógicos que en proyectos anteriores no habían sido desarrollados con tanta fuerza, como la planeación, la integración de los padres y de la comunidad académica, los cuales vislumbraron otros ideales metodológicos de esta estrategia y promovieron espacios para la reflexión y resignificación de concepciones acerca del proceso de enseñanza-aprendizaje, el papel de los niños y de los maestros, la construcción de conocimiento, entre otros.

\section{La planeación}

Buscando que el desarrollo del proyecto fuera un escenario donde se escucharan las voces de todos sus actores, se abrieron espacios para que las expectativas e intereses de cada uno se tuvieran en cuenta.

La planeación conjunta entre las maestras para visualizar posibles temas, elaborar guías de trabajo, compartir experiencias e integrar a los maestros de los espacios disciplinares específicos ${ }^{1}$, permitió concertar ideas, aportes, recibir sugerencias y estrategias. Surgieron así algunas propuestas como crear la banda, la música, el montaje con teatro, etc., y se elaboró el mapa conceptual $^{2}$ definido en algunas sesiones de encuentro entre las maestras directoras de grupo. Claro está, cada grupo siempre buscó su propia identidad, estaba tácitamente acordado que dentro de cada aula la ejecución de lo planeado debería tener características definidas por sus miembros, y seguramente si se preguntara a dos niños de diferente salón acerca del trabajo en el aula, contarían experiencias de aprendizaje diferentes, puesto que cada uno encontró su propia ruta. Con respecto

\footnotetext{
1 Música, teatro, danzas, tecnología, inglés, educación física, instrumental, entre otros.

2 El mapa conceptual es un trabajo realizado por el grupo de maestras, en el cual se visualizan las posibles rutas a seguir de la temática del proyecto de aula.
} 
al trabajo en el aula, procuramos que la planeación y el desarrollo del proyecto fueran fruto del conjunto de intereses, de los niños y de las maestras, ya que todos hacíamos parte de la construcción. Nuestro papel ha estado enfocado en identificar, recolectar y organizar los aportes y preguntas hechas por los niños, para dar un sentido pedagógico en cada una de las actividades que realizan y de esta manera propiciar espacios para la construcción de conocimiento teniendo en cuenta las dimensiones ${ }^{3}$, convirtiéndose estos intereses no solo en el desarrollo del proyecto sino en el motor de sus propios aprendizajes.

\section{El desarrollo del proyecto}

En el camino recorrido se evidenciaba la vida del circo dentro de cada aula. Con un nombre definido democráticamente por los integrantes, estos se llenaron del espíritu circense con disfraces, vestidos, trajes, pelucas, bolos, aros, zancos, sombreros, narices, guantes, capas, maquillaje, dando paso a nuestra intencionalidad inicial: que el aula se convirtiera en un lugar lúdico, con juego de roles que permitía el estudio de la vida en el circo.

En el proceso permanentemente se hicieron ajustes sobre lo planeado y de manera constante se recibieron aportes de los padres, algunos de ellos involucrados en el proyecto, percibiéndose el deseo semanal por compartir algo nuevo. Al hacer este ejercicio de replanteamiento sobre lo planeado, se fueron creando nuevas posibilidades de orientación, redefiniendo estrategias que no se habían considerado, o retomando y jerarquizando las que más relevancia e interés generaban para hacer efectivos los aprendizajes. Esta dinámica permitió la socialización permanente en diferentes momentos del proceso, intercambiando y mejorando puntos de vista. Así se fueron estructurando las acciones escolares que mejoraron las propuestas de aula, cualificando las prácticas para atender la heterogeneidad de los niños en este ámbito colombiano, y de esta manera lograr un ambiente escolar enriquecido con una convivencia democrática, responsable, reflexiva y amable. Así fuimos identificando que la metodología que se empleaba era flexible, sujeta a cambios y variaciones en un ambiente lúdico donde el aprendizaje se da de manera significativa.

3 Dimensiones planteadas por el Ministerio de Educación Nacional en los lineamientos curriculares de preescolar.
Las tareas de aula que se desarrollaron fueron variadas, activas y participativas, de manera tal que la motivación en los niños les permitió que las realizaran con dedicación. Se plantearon propuestas colectivas para desarrollar, así los niños propusieron las acciones, y las inquietudes personales recordaban los pactos que no se habían cumplido y los ayudaban a cumplir. Se fue evidenciando que los niños estaban deseosos por aprender cada día más. Esto permitió que alrededor del tema de interés las maestras promovieran el desarrollo de pensamiento, la construcción de conocimiento, integrando las dimensiones del niño, otorgando sentido al proyecto en la medida que les resultaba interesante. También se dieron momentos para la reflexión crítica; es el caso de la posición de cada uno frente a la participación en el circo de niños artistas, de familias enteras y de animales; se conoció y discutió cómo los niños estudiaban y trabajaban al mismo tiempo, qué pasaba con los animales que vivían fuera de su hábitat natural, entre otras situaciones.

Fuera del aula no se perdió la motivación acerca de lo que se estuviera trabajando. Las labores, consultas y resolución de guías en la casa se ejecutaron con la información llevada por los niños sin el carácter de obligatoriedad, siendo verdaderos canales de comunicación entre familia y escuela. Lo que se realizaba fuera del aula permitía que los conocimientos trascendieran a otros espacios y que desde allí se enriquecieran, para luego ser traídos de vuelta al salón con nuevas expectativas.

\section{¿Y de los padres qué...?}

Como es bien sabido, el desarrollo de los niños, además de promoverse en la escuela, en este proyecto se estimuló desde múltiples escenarios, en este caso en la familia, la cual cobró gran relevancia y se convirtió en uno más de los protagonistas de este proceso; con su participación activa y sus constantes aportes permitieron que los aprendizajes se construyeran de manera conjunta.

La invitación a los padres a involucrarse en el desarrollo y así lograr un mayor impacto abrió las puertas para que los procesos de enseñanza y aprendizaje se dieran en la interacción con otros; se produjo la creación de lazos que se unieron en un solo esfuerzo, compartiendo saberes, deseos e intereses entre padres, maestros y niños. Desde casa, los padres indagaban sobre cómo 
participar, y enviaban aportes que ellos incluían en la planeación semanal; esto generó mayor motivación y dio gran valor a las actividades cuando les ayudaban a los niños a prepararlas.

A lo largo del periodo escolar brindaron sus aportes involucrándose directamente en las propuestas; es el caso de algunos que compartieron actividades que se ajustaban al personaje o tema de interés que se estuviera trabajando. Una de las madres compartió con todos los niños del nivel talleres de globoflexia y pintucaritas (maquillaje artístico), al igual que una invitación a una de las actividades del festival de verano llamada "Festifantasía". Otros padres interesados en el proyecto nos enviaron la información de una obra teatral que presentaba la Universidad Nacional, a la cual los niños asistieron con padres y hermanos.

Las familias enteras acogieron las invitaciones sugeridas: llevarlos al circo que en el momento visitaba la ciudad o a espectáculos que se presentaron en Maloka (el Circo del Sol), presentaciones relacionadas con el tema, entre otras. "La vida en el circo" propició en cada familia una participación activa donde los aportes, informaciones o inquietudes relacionados con el tema eran enviados al colegio, convirtiéndose el aula de clase en un lugar rico en elementos representativos del circo. Las preguntas que surgían desde casa fueron retroalimentadas con investigaciones, consultas en Internet, enciclopedias, rimas, poesías, adivinanzas, lecturas, diversidad textual que luego se compartía con todo el grupo. Se conjugó entonces una dinámica de escuela-familia en la cual la consulta se constituyó en un pilar fundamental en la estrategia de aprendizajes.

En algunas ocasiones, con motivo del "maletín viajero" ${ }^{4} \mathrm{O}$

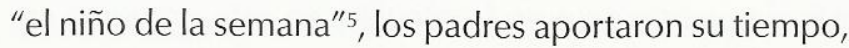
compartiendo iniciativas. En la semana que se trabajaba el rol de payaso, uno de ellos, que fue escogido para ser "el niño de la semana", tuvo un gran respaldo de su familia. Papá y mamá inventaron junto con él una rima, una rutina de payaso, elaboraron títeres de papel para

4 El “maletín viajero" es una estrategia transversal utilizada en el desarrollo del proyecto de aula, en la cual un niño o niña lleva una sorpresa que esté relacionada con el tema de interés y que ayude a responder las inquietudes que van surgiendo.

5 El "niño de la semana" es otra de las estrategias transversales, en la cual el niño o niña escogido debe aportar (en colaboración con sus padres) una actividad como la invitación de un personaje relevante para el proyecto, un juego que involucre los saberes aprendidos, la enseñanza de un dato curioso, entre otros. todos los niños del salón como recuerdo y para cerrar invitaron a un payaso, que se vistió y maquilló frente a ellos para que conocieran cómo se preparaba para la función, en la cual les contó cómo se había convertido en payaso y les mostró la magia del circo valiéndose de chistes y maromas, lo que fue muy motivante para todos; a partir de esta experiencia, en los aportes de los demás niños se fue evidenciando un mayor apoyo de sus familias. Otro de los escogidos para ser el niño de la semana preparó su presentación con el personaje "el malabarista": con su madre y su hermano realizaron una rutina, una presentación de títeres acerca de los malabaristas y los acróbatas. Aunque otros no tuvieron el acompañamiento de sus padres en el colegio, se evidenció el apoyo desde casa en la preparación de las rutinas y vestuario de acuerdo con el personaje. Estos no fueron todos los aportes, hubo muchos más, como invitaciones de circos callejeros, presentaciones de títeres, entre otros.

Los aportes de la familia estuvieron presentes a lo largo de todo el proyecto, algunos con más iniciativas, otros no tanto, pero todos en algún momento tuvieron un espacio de encuentro con sus hijos que les permitió acercarse de una forma diferente y fortalecer sus relaciones familiares.

\section{Integración de los espacios disciplinares específicos}

La estrategia pedagógica global que se maneja en el colegio tiene dos componentes que la constituyen: el proyecto pedagógico de sentido, asumido como el proyecto de aula, y los espacios disciplinares específicos (EDE), que sirven de fortaleza en el desarrollo integral de los niños, desde las diferentes áreas del conocimiento como música, teatro, inglés, danzas, instrumental, tecnología e informática y educación física, proporcionando en forma lúdica el desarrollo de sus capacidades y aptitudes dentro de estos espacios.

La realización conjunta del proyecto en los 5 salones de transición con un mismo interés, permitió que los maestros de los EDE se integraran al trabajo que se estaba realizando; cada uno desde su saber aportó ideas, complementando el trabajo de forma cooperativa y consolidando el equipo de maestros.

Durante 2005, y por mutuo acuerdo tanto de maestras titulares como maestros de los EDE, se propuso como 


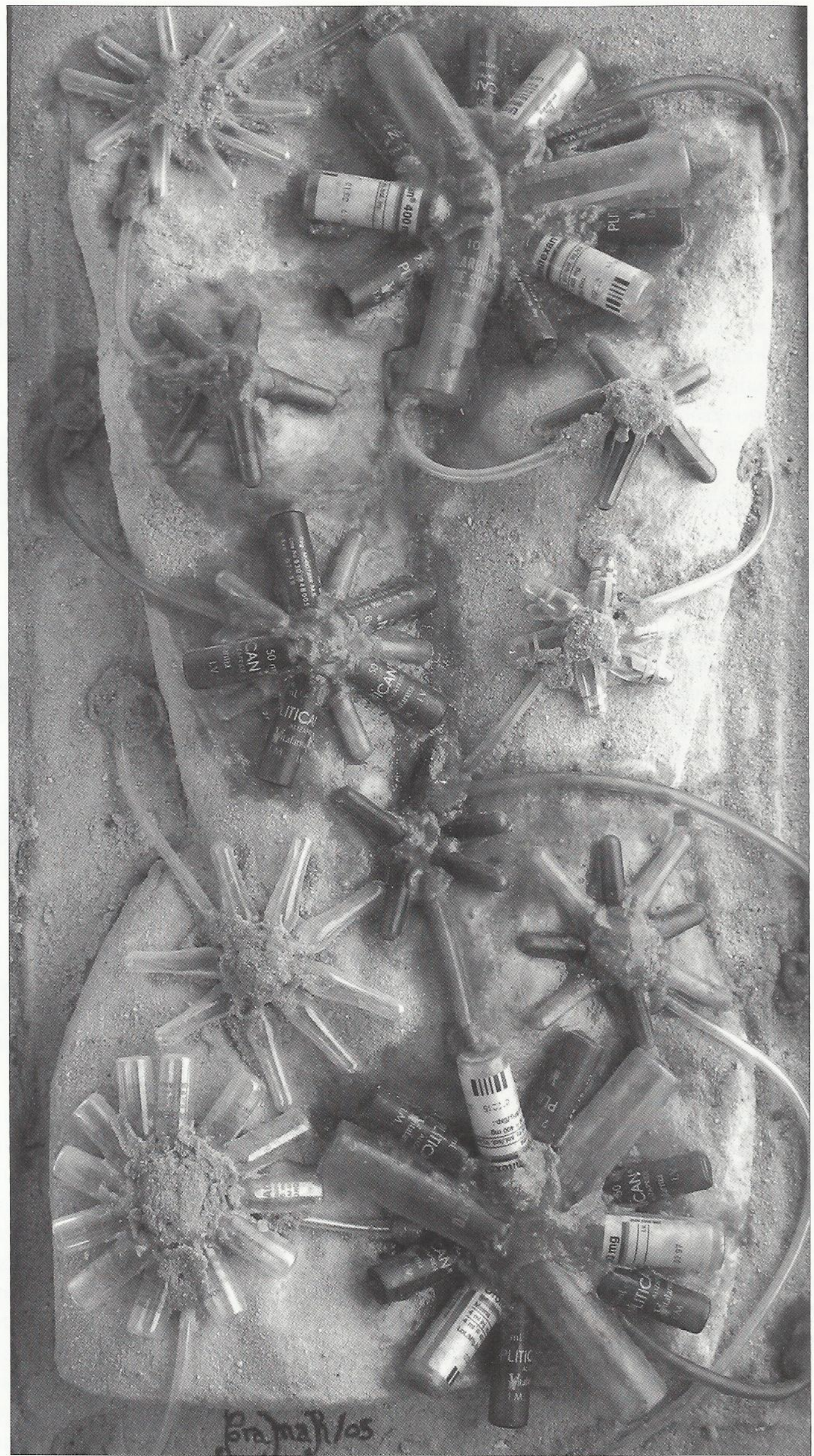

Vilma Graciela Martínez Rivera 》) Flores del mal 2 (Tríptico) 》) Mixta (relieve en papel, ampolletas, sondas, látex, arena) 》) $26,5 \times 49 \times 6 \mathrm{~cm}$
Esta segunda piel de ampolletas y sondas usadas para tratamientos de quimioterapia, irónicamente nos habla de la fuerza de los químicos para matar al tiempo células enfermas y sanas. Con estas flores carnívoras, a muchos cuerpos, antes de sucumbir, les arrancaron el alma supuestamente para dejarlos vivos. 
clausura final la "Puesta en escena de un acto circen$\mathrm{se}^{\prime \prime}$ donde se condensaron las distintas experiencias que profesores y niños hubiesen tenido a través de su paso por estos espacios. Desde cada uno de los EDE se recogió lo trabajado en el aula para retroalimentarlo, o al contrario, lo trabajado en el salón sirvió para darle una nueva ruta. La disposición de los maestros de los EDE fue muy positiva, motivando la socialización de los saberes construidos dentro del aula, que fueron enriquecidos con aportes desde la especialidad de cada uno, facilitando y complementando el desarrollo integral de las temáticas, desencadenándose de esta manera un aprendizaje compartido donde el saber es construido por todos. Así, los niños fueron quienes recibieron toda la influencia artística, teatral, gimnástica, musical, que retroalimenta los conceptos y nuevas construcciones que se complementaron con videos, consultas, lecturas, documentales, charlas espontáneas, exposición de maquetas y diálogos dirigidos.

El apoyo del grupo interdisciplinario del Instituto Pedagógico Nacional fue valioso en los procesos de planeación y realización de la socialización final del proyecto, en el que todos los estudiantes del nivel transición (150 en total) participaron en el montaje y presentación del espectáculo, representando magos, malabaristas, bailarines, acróbatas, taquilleros, banda de bienvenida, música en vivo y protesta de animales con relación al cautiverio, entre otros.

La maestra de música aportó a la puesta en escena "la orquesta del circo" en la que participaron los niños que más se destacaron durante su paso por la clase de música, instrumental y taller de orquesta. Estos espacios tuvieron como objetivo fundamental desarrollar en los niños, de forma lúdica, destrezas técnico-musicales en el ámbito interpretativo, utilizando instrumental Orff ${ }^{6}$, con el fin de optimizar el desempeño de los mismos dentro del grupo musical, en este caso su desenvolvimiento como integrantes fundamentales de la "Orquesta de Transición", con canciones alusivas a personajes que tuvieran conexión directa con el mismo y arreglos vocalinstrumental.

Como propuesta final se realizó un trabajo de creación colectiva donde los niños plasmaron su sentir y conocimiento sobre el tema central del trabajo. Es así como

6 El instrumental Orff está constituido por cajas chinas, triángulos, claves, toc-toc, pandereta (instrumentos de pequeña percusión) y marimbas, xilófonos, metalófonos, sistros o carillones (instrumentos de placa). nace la canción de cierre del acto circense llamada "La diversión del circo", en la cual la letra y la música son de la orquesta de transición de 2005.

La maestra de instrumental se basó en la importancia que jugó la música en el circo antiguo, teniendo en cuenta los diferentes momentos en los que se presentaba la banda: la marcha de bienvenida y de despedida con el desfile de personajes, conformando la banda infantil e integrándose a la presentación final.

La maestra de inglés en sus clases desarrolló estrategias lúdicas con las cuales integró el vocabulario circense, animales, colores, vestuario de los personajes, entre otros; esto permitió a los niños entrar en un aprendizaje contextualizado, rápido, eficaz y significativo. De igual manera este trabajo se integró con el de música en el montaje y ejecución de canciones en inglés y español, con el apoyo del grupo de instrumental, para la puesta en escena.

El profesor de teatro tuvo claro que era básico asentarse en los fundamentos de todo lo que tiene que ver con el espectáculo, sin importar el género, y siendo el circo un género rico y llamativo para los niños, se facilitaron los ensayos sin que los chicos perdieran la motivación. El espectáculo fue integrado por "artistas" del grado de transición, previamente seleccionados según sus capacidades y rendimiento obtenido en experiencias lúdicas a través del año escolar. Se dispuso de suficiente cantidad y calidad de intérpretes infantiles para poder desarrollar eficientemente el espectáculo; fue así que se permitió la participación de la totalidad de los niños del nivel, logrando la aceptación de ellos y del público que asistió a un montaje rico en ambientes, integrando el elenco en situaciones que enriquecieron nuestro proyecto pedagógico y generando en los niños procesos de participación activa.

El profesor de educación física se integró al proceso teniendo en cuenta el desarrollo de las habilidades y capacidades físicas de los niños, las pautas principales estaban enmarcadas en la expresión corporal por medio de ejercicios y movimientos con elementos de gimnasia escolar. Desarrollaron esquemas con cualidades motrices que, acompañados de música, aportaron al montaje final las acrobacias y malabares, utilizando barras de equilibrio, paralelas, bancas y vigas, realizando una combinación entre fuerza y equilibrio. Igualmente danzas realizó su aporte al montaje con la participación de un grupo de niños que representaron bailes que se 
ajustaron a las nuevas producciones en actos circenses como los del "circo del sol".

\section{Lo que aprendimos}

Este proyecto fue valioso para los niños, los padres y los maestros; todos aprendimos cada día más acerca de la vida en el circo, enriquecimos nuestras prácticas y nos divertimos. Algunos de los logros en el trabajo pedagógico fueron: el replanteamiento de estrategias que ayudaron al acercamiento a la lengua escrita y a la lectura de una forma espontánea y lúdica, lo cual produjo tanto la construcción de conocimiento como la posibilidad de socializar y divertirse con el lenguaje.

De manera natural y espontánea, los niños, además de involucrar en su vocabulario palabras correspondientes al tema dándoles un significado, construyeron su propio discurso acerca de la vida en el circo desde su vivencia con esta experiencia y el conocimiento construido de manera reflexiva; además de fortalecer su autoestima por medio de la socialización de saberes, se sintieron importantes y capaces cuando compartieron con otros sus conocimientos o en las oportunidades en que trabajaron en grupo.

Las actividades permitieron a los niños desarrollar su capacidad simbólica, representando con gran facilidad los personajes, destacando de ellos sus características principales, pero siempre poniendo un toque de sí mismos. Se dieron oportunidades para hacer comparaciones y mediciones a partir de las cuales realizamos registros que incluyeron la medición de la altura de los animales y su longevidad, aspectos que impactaron a los niños.

La posibilidad que brindó el proyecto a los niños de expresar sus sentimientos y emociones les permitió acercarse de una forma más humana a situaciones como el cautiverio de los animales, y las implicaciones que tiene integrarlos en actos circenses, expresando con asombro afirmaciones como "cuando están encerrados no pueden tener bebés" o "a los leones no les puede crecer la melena si no están en su hábitat".

Las relaciones que se establecieron entre las maestras y los niños fueron interactivas, en el aula no había uno que supiera más que otro, sino al contrario: los aportes que cada uno iba dando, a partir de su experiencia, fortalecían las intervenciones de sus compañeros o las de los adultos. La posibilidad de participación auténtica les dio paso a los niños para involucrarse al proyecto de manera real, consciente, voluntaria e interesada en la satisfacción de logros e intereses personales como grupales, y se amplió el espectro de consulta con los intereses individuales haciendo que el trabajo diario fuera dinámico, agradable y motivador.

Gracias a la permanente contextualización surgieron los aprendizajes de forma espontánea como una necesidad, logrando que los procesos de pensamiento se desarrollaran de manera mágica, como fue el caso de la lectura y la escritura, las cuales los niños construyeron según su necesidad, algunas veces con mediación de las maestras y otras sin acompañamientos. El trabajo por proyectos nos permitió resaltar en los niños más sus fortalezas que sus debilidades, respetando su ritmo de aprendizaje, evidenciándose de una forma más clara los avances.

Abrir el espacio para que la familia esté presente en el proceso de aprendizaje de sus hijos durante el desarrollo del proyecto fue una fuente de alimentación permanente, ya que se enriqueció constantemente con la diversidad de los aportes; además de fortalecer los lazos afectivos entre ellos, se optimizaron los procesos de comunicación, y la consulta y las actividades en casa se convirtieron en una excusa para crear espacios que permitieron compartir en familia.

Escribir sobre esta experiencia nos permitió realizar un ejercicio de pensamiento, producto de un proceso sistemático de reflexión generado desde nuestra participación en la RED-CEE, con base en las acciones educativas, y de cómo hemos gestado la construcción de pensamiento a partir de esta estrategia pedagógica. La fundamentación ha surgido de la experiencia empírica, la cual hemos ido enriqueciendo con nuevos saberes por medio de visitas y acercamientos a otros colegios, participación en seminarios, asistencia a conversatorios sobre pedagogía de proyectos, entre otros. Todo esto tiene como fin el beneficio de la cualificación personal y grupal.

El grupo que consolidamos entre las maestras nos permitió llevar a discusión las experiencias que ocurrieron en cada salón, enriqueciendo nuestra mirada individual, y tomando elementos que tuvieron éxito para proponerlos a nuestros grupos, favoreciendo su desarrollo y fortaleciendo las estrategias propias del proyecto de aula. Además nos permitió reflexionar y autoevaluar, para 
avanzar y mejorar la práctica educativa $y$ hacer evidentes otros principios que antes no lo eran, como es el caso de la planeación.

La integración de los diferentes EDE al proyecto permitió consolidar un trabajo interdisciplinario con todos los maestros del nivel de transición, ofreciendo ambientes favorables donde los niños socializaron saberes y conocimientos con sus pares, facilitando y complementando la construcción integral de los aprendizajes.

Tenemos muy claro que estos cambios se irán cualificando y perfeccionando por medio del aprendizaje constante de los procesos vividos en la escuela. Nuestra búsqueda no termina aquí, estamos en el camino de continuar construyendo y consolidando nuevos conocimientos, no solo acerca de la estrategia de proyecto de aula, sino de todo lo que la educación implica: una constante reflexión de las prácticas, claro está, acompañada de la producción de saberes pedagógicos, a fin de desarrollar una perspectiva teórica para interpretar las propuestas contemporáneas de las cuales estamos constantemente bombardeados, preguntándonos de allí los cómo, por qué, para qué, quiénes, cuándo, dónde, otorgándole un espacio al ejercicio de la pedagogía.

\section{Referencias}

BRUNER, Jerome. 1984. "Juego, pensamiento y lenguaje". En: Acción, pensamiento y lenguaje. Madrid: Alianza Editorial.

GARTON, F. Alison. 1994. Interacción social y desarrollo del lenguaje y la cognición. Barcelona: Paidós.

HERNÁNDEZ, Fernando y VENTURA, Monserrat. 1998. La organización del currículum por proyectos de trabajo. s. p. i.

ROGOFF, Barbara. 1984. Aprendices del pensamiento. El desarrollo cognitivo en el contexto social. Barcelona: Paidós.

\section{Diálogo del conocimiento}

La experiencia "La vida en el circo" que nos comparten las profesoras del Instituto Pedagógico Nacional, sin duda constituye un aporte valioso a la innovación y creatividad en el aula. Una vez más, es de destacar la importancia que tiene el ejercicio de sistematizar, que en este caso nos brinda la posibilidad de aprender de la reconstrucción que las autoras hacen de su experiencia.

Las reuniones semanales en las instituciones educativas, que pueden convertirse en áridas rutinas, aquí fueron aprovechadas para socializar las experiencias de cada maestra. Esta socialización las llevó a la construcción de un proyecto de aula, que tuviese una temática interesante, significativa y divertida tanto para los niños como para las maestras: “La vida en el circo". Así, en marzo de 2005 emprendieron un viaje fantástico con los niños y las niñas de los grados de transición. Me parece de especial importancia rescatar varios puntos:

La experiencia surge de la sentida búsqueda de un grupo de maestras que se proponen enriquecer la cotidianidad de su aula de preescolar incursionando en el terreno de la creatividad y se nutre de una reflexión que se apoya en los saberes previos. Toma especial importancia el desarrollo de principios pedagógicos no trabajados hasta el momento en su institución.

Se establece una interacción con diferentes teorías pedagógicas desde lo vivido en el aula, lo cual permite una conversación inspirada en lo realizado y lo realizable.

El sentir, los deseos y las propuestas de los niños tuvieron un lugar protagónico en el proyecto.

En el desarrollo del proyecto hay una clara contextualización del conocimiento de manera concreta a través de la figura no solo del circo, sino de la Vida que lo habita.

El circo toma el carácter de "escenario" en el que las artes y el juego se constituyen en los lenguajes para generar actividades, realizaciones y por tanto aprendizajes.

Se logra una integración de los padres y de la comunidad académica por medio de espacios para la reflexión y resignificación de concepciones acerca del proceso de enseñanza-aprendizaje, el papel de los niños y de los maestros, la construcción de conocimiento, entre otros.

Se logra una integralidad disciplinar que proporciona nuevos sentidos a los contenidos de enseñanza-aprendizaje y que pone la experiencia en la vía de los currículos emergentes.

El proceso da muestra de la toma de conciencia de las maestras sobre su propio aprendizaje y del proceso de enseñanaza-aprendizaje de sus alumnos al formular acciones formativas necesarias para alcanzar los objetivos y metas en los procesos de concreción de construcción del conocimiento.

Se hace una valoración de lo aprendido con esta experiencia y de la manera en que fue aprendido.

Asimismo, se alcanza un cuidadoso proceso de diseño, planeación y desarrollo del proyecto con la participación de un amplio grupo de actores educativos. Por medio de una dinámica de construcción colectiva los docentes integraron lo planeado al aula transformando la realidad con sus alumnos y dando nuevos significados a la infancia, a ser maestro y a la escuela. 